\title{
A Case Study on Microbial Diversity Impacts of a Wastewater Treatment Plant to the Receiving River
}

\author{
Hongfei Zhu*, Bailian Li, Ning Ding, Zheng Hua, Xiaoxu Jiang \\ Department of Environmental Science and Engineering of Liaoning Technical University, Fuxin, China \\ Email: ^hongfei.zhu@yahoo.com
}

How to cite this paper: Zhu, H. F., Li, B. L., Ding, N., Hua, Z., \& Jiang, X. X. (2021). A Case Study on Microbial Diversity Impacts of a Wastewater Treatment Plant to the Receiving River. Journal of Geoscience and Environment Protection, 9, 206-220. https://doi.org/10.4236/gep.2021.94013

Received: March 17, 2021

Accepted: April 24, 2021

Published: April 27, 2021

Copyright ( 2021 by author(s) and Scientific Research Publishing Inc. This work is licensed under the Creative Commons Attribution International License (CC BY 4.0).

http://creativecommons.org/licenses/by/4.0/

\begin{abstract}
The discharge from wastewater treatment plants (WWTPs) into the receiving river is of concern for the health of the ecosystem. In this study, the microbial diversity impact of Fuxin Meng (FM) WWTP was completed with three pairs of primers. At the time of impact assessment, biochemical tests were carried out as well to investigate the water quality downstream and upstream FMWWTP. The general results showed this plant increased downstream biochemical oxygen demand (BOD) and chemical oxygen demand (COD) by 4.3- and 8.1-fold respectively, in comparison with the upstream. The microbial diversity impact from the plant was also investigated with three pairs of universal primers targeting rRNA genes and the internal transcribed spacer (ITS) by high-throughput sequencing. The downstream diversity of the bacterial and fungal communities was generally higher than upstream, with the exception of the eukaryotic community. Both biochemical and microbial assessments suggested that FMWWTP deteriorate Xi River water quality, potentially acting as a pollutive source. Of the receiving water. Accordingly, it was necessary to improve the operation of WWTP to live up to the environmental quality standards.
\end{abstract}

\section{Keywords}

rRNA Sequencing, Microbial Community, Impact, Primer, Wastewater

\section{Introduction}

Discharge from WWTPs often disturbs the natural microbial communities of the receiving waterbodies. The impact caused by WWTP on the microbial communities of the river is of warm concern for environmental management. A case study was fulfilled to reveal the impact of a WWTP on microbial communi- 
ties using community-specific primers, along with biochemical tests for assistance. Xi River, the receiving water of FMWWTP located in Fuxin, Liaoning, China $(42.052334 \mathrm{~N}, 121.747798 \mathrm{E})$. The city had developed centering the middle axis, $\mathrm{Xi}$. Therefore, the maintenance of the river was of significance for the city due to its mother-river role. The river sides had long been recreational places and its water source generously nourished the people, agriculture and industry. Just because of this, close attention had been paid to the river ecosystem by the locals. The urban environmental protection administrations microbiologically surveilled the river water to assess the extent of pollution for management guidance (Frigon et al., 2013). Studying the microbial diversity of river-water may help to improve the wastewater treatment facilities or to identify any healththreatening factors.

Wastewater effluents may contribute to water source pollution by loading nutrients into the receiving water. In addition, the microbes residing in the WWTP may flow out with the effluent current, which may disturb the microbial community of the river. Therefore, inadequate wastewater treatment may endanger the health of the aquatic ecosystem and cause diseases. Although chemical and biochemical testing, such as of total nitrogen (TN), total phosphorus (TP), biochemical oxygen demand (BOD), and chemical oxygen demand (COD), is conventionally available, few analyses had been performed on the microbial community using sequencing method for Xi. High-throughput sequencing has been irreplaceable as an emerging technology and greatly exceeds the reach of the regular methods used for water quality control. DNA sequencing makes it possible to reimagine water quality monitoring as it can provide more detailed and important information for Xi River management.

Microbial community composition partially reflects chemical parameters such as $\mathrm{pH}$ and pollutant concentrations. Therefore, it is necessary to promptly investigate the microbial diversity of the Xi River water subjected to the FMWWTP discharge (Yotova et al., 2019). In this study, river water samples were taken from sites both upstream and downstream of the outlet. The prokaryotic and eukaryotic microbial community diversities of the samples were surveyed by sequencing the $16 \mathrm{~S}$ and $18 \mathrm{~S}$ rRNA genes, respectively. This sequencing-based method of conserved genes is independent of culturing or microscopy. It is convincible that multiple primer sets provide a good solution for determining microbial community structure (Blackwood et al., 2005; Wang et al., 2018). In order to obtain an enhanced description of the microbial community structure and diversity of $\mathrm{Xi}$, three universal pairs of primers were adopted to target different kingdoms for the assessment. Meanwhile, the other purpose of the design was to determine if the primer-specific microbial assessment corresponded with the biochemical assessments. The present analysis of the microbial communities was conducted with the aim of assessing the impact caused by the plant.

To the best of our knowledge, this is the first report to profile the Xi River microbial community using high-throughput sequencing technology. The results of the study may be conducive to upgrading the wastewater treatment processes of 
the FMWTP.

\section{Materials and Methods}

\subsection{Sampling and Water Quality Testing}

The water samples were obtained from Xi River, Fuxin, Liaoning, China in the first week of May, 2018. The two sampling sites were $500 \mathrm{~m}$ upstream and $500 \mathrm{~m}$ downstream of the water outlet of FMWTP. The water samples were taken from approximately $30-50 \mathrm{~cm}$ below the water surface using 12 sterilized glass bottles with $1 \mathrm{~L}$ capacity. The samples were immediately transported to the laboratory in an icebox with a constant temperature of approximately $4^{\circ} \mathrm{C}$.

The water quality testing complied with the GB3838-2002 regulations (environmental quality standards for surface water). Temperature and $\mathrm{pH}$ were measured at the two sampling sites. The $\mathrm{BOD}_{5}, \mathrm{COD}, \mathrm{TP}, \mathrm{TN}$, and ammonia nitrogen (AN) were measured immediately after sample collection in the laboratory using the methods recommended in GB3838-2002 (Shi et al., 2020; Ye \& Kameyama, 2020; Yotova et al., 2019).

\subsection{Total DNA Extraction of Water Samples for Community Analysis}

Approximately $10 \mathrm{~L}$ of fresh water sample from the Xi River was filtered through membranes with pore sizes of $0.22 \mu \mathrm{m}$ to collect the bacterial and eukaryotic microorganisms until visible pellets were observed on the upper side of the membrane. Total bacterial DNA was extracted from the SB samples, using Bacteria Genomic DNA Extraction Kits (Tiangen Biotech Co., Ltd., Beijing). The genomic DNA for eukaryotic and fungal samples (SF and SFF) was extracted using Fungal Genomic DNA Extraction Kits (Tiangen). The extraction operation was performed following the manufacturer's protocol.

The bacterial DNA samples from the upstream (SB1) and downstream (SB2) water sampling locations were amplified using a primer pair targeted to the $16 \mathrm{~S}$ rRNA gene (27F 5'-AGAGTTTGATCCTGGCTCAG-3' and 338R 5'-TGCTGCCTCCCGTAGGAGT-3') (Ravel et al., 2011). The 27F/338R primer set was used to garner common bacteria residing in the water. The eukaryotic samples from the upstream (SF1) and downstream (SF2) water sampling locations were amplified using a primer pair targeted to the 18S rRNA gene (Euk1391F 5'-GTACACACCGCCCGTC-3' and EukBR 5'-TGATCCTTCTGCAGGTTCACCTAC-3') (Adams et al., 2013; Amaral-Zettler et al., 2009; Begerow et al., 2010). The forward primer, Euk1391F, is a universal primer, while EukBR is a reverse primer that favors eukaryotes; these primers were used to form a combination strategy for amplification. Finally, the fungal samples from the upstream (SFF1) and downstream (SFF2) water sampling locations were amplified using a primer pair targeting the ITS region (ITS1F

5'-CTTGGTCATTTAGAGGAAGTAA-3' and ITS2R

5'-GCTGCGTTCTTCATCGATGC-3'). The primer set ITS1F/ITS2R has already been shown to provide good resolution with regard to fungal identification $(\mathrm{Gu}-$ 
arro et al., 1999; Reblova et al., 2013).

The PCR products were quantified using QuantiFluor ${ }^{\mathrm{TM}}$-ST (manufactured by Promega) for sequencing library preparation. For Illumina Miseq ${ }^{\circledR}$ sequencing, adapters were linked to the fragments to be sequenced via the PCR method. Single-stranded DNA fragments were obtained from denaturing. The entire protocol was conducted following the instructions provided with the TruSeqTM DNA Sample Prep Kit (Illumina, Inc.). Shanghai Majorbio Company was entrusted to conduct the sequencing using the Illumina Hiseq $\times$ Ten Platform. Paired-end reads from the Miseq system were assembled using overlapping. OTU clustering analysis was carried out, which paved the way for in-depth data mining.

\subsection{Data Processing and Bioinformatic Analysis}

The bacteria, eukaryotes, and fungi present in the samples were allocated to phyla, orders, families, genera, and species by aligning the obtained sequences with public databases (Yin et al., 2014). The raw data were processed using Mothur and QIIME (Quantitative Insights Into Microbial Ecology) software to filter out sequences that displayed 5' mismatch, ambiguous bases, or more than eight homologous bases, or that were shorter than $150 \mathrm{bp}$. Chimeras were removed using the uchime method of Mothur. Sequences were clustered into OTUs using USEARCH (version 7.0; http://drive5.com/uparse/) using an identity threshold of $97 \%$. " $p<0.01$ " was set as the default and used for all of the analyses.

Software and algorithms were derived from the databases of Ribosomal database Project (RDP; Release 11.1; http://rdp.cme.msu.edu/), Silva (Release 128; http://www.arb-silva.de), Greengene (Release 13.5; http://greengenes.secondgenome.com/), Unite (Release 6.0; http://unite.ut.ee/index.php), and FDR (False Discovery Rate). A confidence coefficient value of 0.7 was used in QIIME (http://qiime.org/scripts/assign_taxonomy.html) and RDP Classifier (version 2.2; http://sourceforge.net/projects/rdp-classifier/). R computer language was employed to build the charts on the i-sanger platform (Ghilamicael et al., 2018).

\subsection{Community Diversity Analysis}

Alpha diversity index analysis was performed on the basis of the sequencing data and largely reflected the richness and abundance of the species within the community. The Shannon, Simpson, and Chao indexes and coverage values were used to describe the communities and their diversity (Zhao et al., 2014).

\section{Results and Discussion}

\subsection{Biochemical Assessment of Water Quality}

The overall quality of the upstream sampling site was superior to that of the downstream site according to GB3838-2002. FMWWTP was thus preliminarily predicted to impact its receiving water. All water quality data that were collected are shown in Table 1 . The measured $\mathrm{pH}$ and $\mathrm{TP}$ values complied with the limits 
Table 1. Quality parameters of water samples from upstream and downstream sampling sites.

\begin{tabular}{cccccccc}
\hline Sites & Temperature $\left({ }^{\circ} \mathrm{C}\right)$ & $\mathrm{pH}$ & $\mathrm{BOD}_{5}(\mathrm{mg} / \mathrm{L})$ & $\mathrm{COD}(\mathrm{mg} / \mathrm{L})$ & $\mathrm{TP}(\mathrm{mg} / \mathrm{L})$ & $\mathrm{TN}(\mathrm{mg} / \mathrm{L})$ & $\mathrm{AN}(\mathrm{mg} / \mathrm{L})$ \\
\hline Upstream & $11.4 \pm 0.1$ & $6.8 \pm 0.1$ & 10 & 12.5 & 0.079 & 24.93 & 3.669 \\
Downstream & $10.9 \pm 0.1$ & $6.9 \pm 0.1$ & 43 & 100 & 0.242 & 25.13 & 0.524 \\
\hline
\end{tabular}

set by the authorities. The temperature $\left(10.9^{\circ} \mathrm{C}-11.4^{\circ} \mathrm{C}\right)$ and $\mathrm{pH}(6.8-6.9)$ were similar for both sampling sites. The TP concentration was $0.079 \mathrm{mg} / \mathrm{L}$ upstream and $0.242 \mathrm{mg} / \mathrm{L}$ downstream; both of these values were in compliance with the required standard. The TN at both sampling sites $(24.93 \mathrm{mg} / \mathrm{L}$ upstream and $25.13 \mathrm{mg} / \mathrm{L}$ downstream) heavily exceeded the maximum limit set $(2.0 \mathrm{mg} / \mathrm{L})$. Upstream AN $(3.669 \mathrm{mg} / \mathrm{L})$ was also in excess of the maximum standard (2.0 $\mathrm{mg} / \mathrm{L}$; GB3838-2002 for an unknown reason. Fortunately, the downstream AN $(0.524 \mathrm{mg} / \mathrm{L})$ was within the limit. The upstream $B B_{5}$ value of $10 \mathrm{mg} / \mathrm{L}$ and COD value of $12.5 \mathrm{mg} / \mathrm{L}$ increased to $43 \mathrm{mg} / \mathrm{L}$ and $100 \mathrm{mg} / \mathrm{L}$, respectively, downstream. The $\mathrm{TN}, \mathrm{BOD}_{5}$, and $\mathrm{COD}$ at both sites indicated that the plant did not adequately treat wastewater. Therefore, FMWWTP potentially acted as a pollutant of the receiving waterbody. The inadequate treatment of wastewater may lead to a large amount of nutrients being loaded into Xi River, resulting in reduced water quality. The microbial communities were expected to shift in response to the changes in nutrient concentrations between the upstream and downstream sites. Upgrading aspects of the WTP, such as running costs, operator skills, and infrastructural maintenance, is expected to contribute to improving the standard of the discharge.

\subsection{Primer-Specific OTU Clustering and Alpha Diversity Analysis}

For the bacterial samples, SB1 and SB2, 39259 (12,464,732 bp) and 52370 $(16,840,094 \mathrm{bp})$ optimum reads were obtained respectively, using $27 \mathrm{~F} / 338 \mathrm{R}$ to amplify the V1-V2 region. In total, 783 OTUs (or taxa) were identified through the sequence alignment with the five public databases. Similar OTUs were clustered if similar threshold used. There were 318 OTUs that were shared by both SB1 and SB2, and 442 and 23 OTUs were unique to SB2 and SB1, respectively. The Chao index for SB2 reached 764.43, which was much higher than that of SB1 (420.22), which proved that the bacterial community of the former was more species-rich. Moreover, the Shannon (SB1, 3.58 and SB2, 5.01) and Simpson indexes (SB1, 0.05 and SB2, 0.02) indicated that the diversity of the SB2 community was greater than that of SB1. The shifts in the bacterial community reflected the difference in water quality between the two sampling sites.

From the amplification of the samples SF1 and SF2 using the primer pair Euk1391F/EukBR, 67012 and 32856 sequences were obtained corresponding to 304 and 212 OTUs, respectively. There were 116 OTUs that were common to both SF1 and SF2, while 118 and 96 OTUs were unique to SF1 and SF2, respectively. The Chao index for SF2 was 225.77, indicating that the species richness was lower than in SF1 (Chao index 309.71). SF1 had a lower Simpson's index 
(0.17) and higher Shannon index (2.44) than SF2 (Simpson of 0.22 and Shannon of 2.32), indicating that the diversity of the eukaryotic community was higher in SF1 than in SF2. The diversity trends observed for the SF samples were opposing those observed for SB.

From the amplification of the SFF samples using the ITS1F/ITS2R primer pair, 40012 (SFF1) and 41004 (SFF2) sequences were clustered into 212 and 246 OTUs, respectively. Of these OTUs, 141 were common to both SFF1 and SFF2. The Chao index value of SFF2 (250.04) was higher than that of SFF1 (216), which indicated that the fungal community of SFF2 was more species rich. The Shannon (SFF1, 2.64 and SFF2, 1.90) and Simpson's (SFF1, 0.17 and SFF2, 0.28) indexes indicated that the fungal community diversity was higher in SFF2 than in SFF1. Thus, the fungal community diversity in the downstream sample was higher than in the upstream; this trend was also observed in the prokaryotic analysis (SB samples). The analysis of the SFF samples once again indicated that the downstream sampling site was subjected to pollution. The coverage of all the samples was near $100 \%$, which indicated that the libraries and sequencing data were matching. The overall statistics of the sequencing information and alpha diversity indexes are summarized in Table 2 . With respect to the trends in alpha diversity between the upstream and downstream sites, the impacts caused by the FMWWTP differed for the bacterial, eukaryotic, and fungal communities.

\subsection{Genus-Level Bacterial and Eukaryotic Diversity}

At the genus level, the bacterial community of SB1 was less complicated than that of SB2 (Figure 1). The genus "norank_c_Cyanobacteria" (3402 OTUs in SB1 and 3032 OTUs in SB2) was a rich component of both water samples, while the other dominant genera varied between samples. Cyanobacteria, which are the most common phytoplanktonic organisms associated with eutrophication of fresh water systems, can reduce water transparency, and cause oxygen shortages and cyanotoxin production (Rapala et al., 2006). Close attention should be paid to the potential threats of a Cyanobacteria bloom. In accordance with these data,

Table 2. Operational taxonomic unit (OTU) clustering and analysis of alpha diversity indexes.

\begin{tabular}{|c|c|c|c|c|c|c|c|c|c|c|c|c|}
\hline \multirow{2}{*}{$\begin{array}{l}\text { Sample } \\
\text { name }\end{array}$} & \multicolumn{7}{|c|}{ OTU clustering and classification } & \multicolumn{5}{|c|}{ Alpha diversity indexes } \\
\hline & Sequences & OTU number & Phyla & Classes & Orders & Families & Genera & Sobs & Shannon & Simpson & Chao & Coverage \\
\hline SB2 & 41750 & 760 & 26 & 51 & 103 & 182 & 300 & 760 & 5.01 & 0.02 & 764.43 & 0.99 \\
\hline SF1 & 67012 & 304 & 23 & 37 & 47 & 59 & 76 & 304 & 2.44 & 0.17 & 309.71 & 0.99 \\
\hline SF2 & 32856 & 212 & 27 & 40 & 53 & 66 & 87 & 212 & 2.32 & 0.22 & 225.77 & 0.99 \\
\hline SFF1 & 40012 & 212 & 6 & 14 & 23 & 29 & 34 & 212 & 2.64 & 0.17 & 216 & 0.99 \\
\hline SFF2 & 41004 & 246 & 7 & 17 & 29 & 41 & 48 & 246 & 1.90 & 0.28 & 250.04 & 0.99 \\
\hline
\end{tabular}

Note: “1” represented upstream, "2" for the downstream samples. 


\begin{tabular}{|c|c|c|}
\hline 175 & 138 & Brevundimonas \\
\hline 89 & 110 & norank_f_NS11-12_marine_group \\
\hline 221 & 336 & norank_f__Hydrogenophilaceae \\
\hline 499 & 55 & Pedobacter \\
\hline 309 & 143 & Hydrogenophaga \\
\hline 382 & 155 & Fluviicola \\
\hline 391 & 112 & Candidatus_Aquirestis \\
\hline 58 & 262 & Thermomonas \\
\hline 0.1 & 299 & OM60_NOR5_clade \\
\hline 1 & 286 & norank_o_SC-I-84 \\
\hline 17 & 268 & unclassified_k_norank \\
\hline 9 & 262 & Pseudomonas \\
\hline 6 & 231 & Tabrizicola \\
\hline 3 & 253 & norank_f_mle1-27 \\
\hline 4 & 242 & norank_o_Xanthomonadales \\
\hline 44 & 178 & norank_f_NS9_marine_group \\
\hline 9 & 213 & norank_f_Comamonadaceae \\
\hline 13 & 193 & Zoogloea \\
\hline 2 & 494 & norank_f_SJA-28 \\
\hline 1 & 491 & norank_c_OM190 \\
\hline 1 & 459 & BD1-7_clade \\
\hline 10 & 449 & Rhizobacter \\
\hline 63 & 410 & Chitinivorax \\
\hline 9 & 423 & norank_p__Parcubacteria \\
\hline 12 & 404 & Dokdonella \\
\hline 4 & 397 & Nannocystis \\
\hline 46 & 670 & norank_f_Xanthomonadaceae \\
\hline 6 & 652 & norank_f_Cytophagaceae \\
\hline 7 & 635 & Nitrosomonas \\
\hline 12 & 966 & Sulfuritalea \\
\hline 15 & 817 & OM27_clade \\
\hline 12 & 779 & Ferruginibacter \\
\hline 5 & 4075 & Nitrospira \\
\hline 144 & 3444 & norank_f_Saprospiraceae \\
\hline 154 & 1958 & Thauera \\
\hline 20 & 1672 & Terrimonas \\
\hline 22 & 1361 & Dechloromonas \\
\hline 236 & 967 & Leptothrix \\
\hline 545 & 1129 & unclassified_f_Comamonadaceae \\
\hline 672 & 948 & Simplicispira \\
\hline 1505 & 120 & unclassified_f_Sporichthyaceae \\
\hline 1246 & 128 & hgeI_clade \\
\hline 1876 & 315 & unclasssified_f_Rhodobacteraceae \\
\hline 1650 & 912 & Arenimonas - \\
\hline 1621 & 615 & Aquabacterium \\
\hline 1428 & 614 & Flavobacterium \\
\hline 1439 & 424 & Limnohabitans \\
\hline 1335 & 504 & Albidiferax \\
\hline 3402 & 3032 & norank_c_Cyanobacteria \\
\hline 4784 & 662 & unclassified_o__Cytophagales \\
\hline ही & हु' & \\
\hline
\end{tabular}

(a)

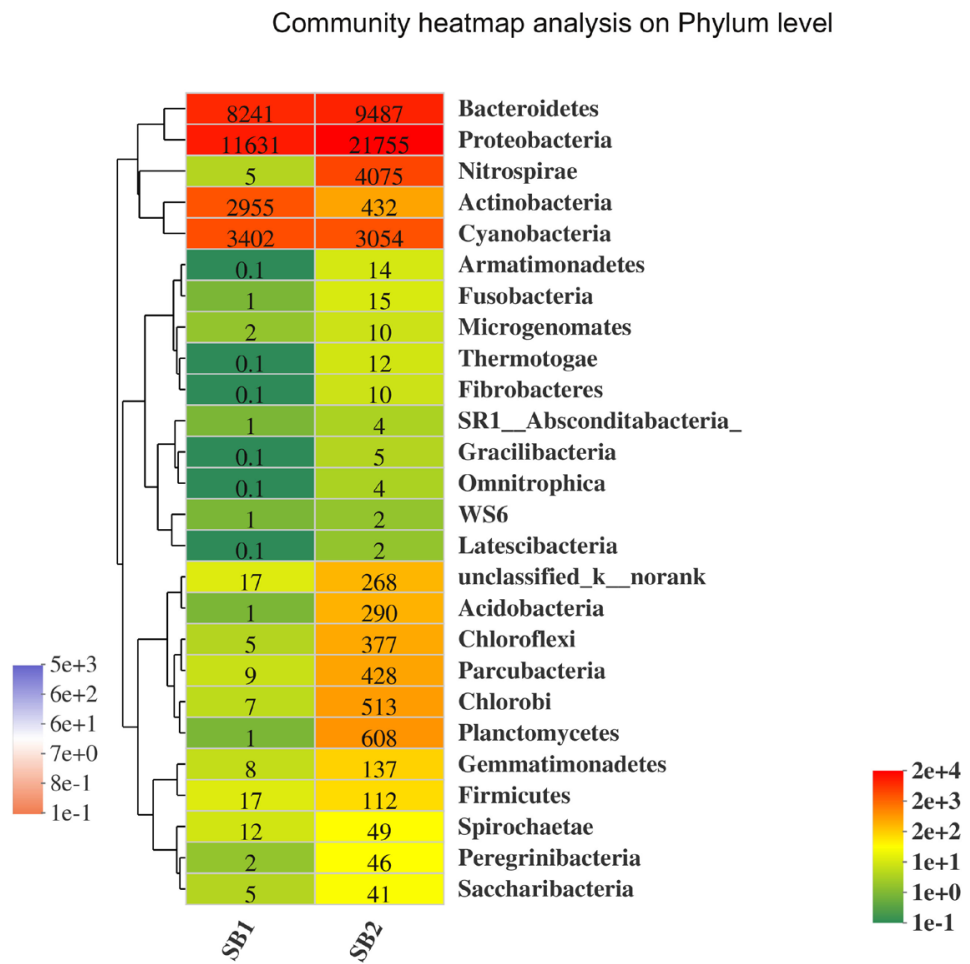

(b)

Figure 1. Heatmap of the bacteria identified at the genus-(a) and phylum-level (b) from samples collected upstream (SB1) and downstream (SB2) of the discharge released from Fuxin Meng Wastewater Treatment Plant into the Xi River.

the mixing of upstream water with the FMWWTP discharge did not significantly alter the phototrophic function of Cyanobacteria or shift the river cyanobacterial community at the downstream site. The decreased abundance of "norank_c_Cyanobacteria" OTUs in the downstream sample may have resulted from water dilution.

"Unclassified_o_Cytophagales" (4784 OTUs),

"Unclassified_f_Rhodobacteraceae" (1876 OTUs), Arenimonas (1650 OTUs), Aquabacterium (1621 OTUs), "Unclassified_f_Sporichthyaceae" (1505 OTUs), Limnohabitans (1439 OTUs), Flavobacterium (1428 OTUs), Albidiferax (1335 OTUs), Pedobacter (499 OTUs), and the HGCL-clade (1246 OTUs) were more abundant in SB1 than in SB2. Most of the core genera were closely related with the processes of organic substance degradation or denitrification. Only 9.7\% (abundance percentage) of the genera in SB1 were unclassified. 
A higher diversity was observed at the genus level in the SB2 bacterial community. The abundances of Nitrospira (4075 OTUs), "Norank_f_Saprospiraceae" (3444 OTUs), Thauera (1958 OTUs), Dechloromonas (1361 OTUs) Terrimonas (1672 OTUs), Leptothrix (967 OTUs), "Unclassified_f_Comamonadaceae” (1129 OTUs), Sulfuritalea (966 OTUs), Simplicispira (948 OTUs), OM27_clade (817 OTUs), "Unclassified_o_Cytophagales" (662 OTUs) and Ferruginibacter (799 OTUs) were higher in SB2 than in SB1. Nitrospira species, which are commonly found in terrestrial or aquatic environments, include a suite of microorganisms that are responsible for ammonia or nitrite oxidizing (Pjevac et al., 2017). The core bacterial genera in SB2 mainly function in the process of nitrification, denitrification, and organic substance metabolism. In the process of water treatment, ammonia-oxidizing, nitrifying, and other heterotrophic or autotrophic denitrifying bacteria remain in the treated water (Huang et al., 2010).

With respect to the eukaryotic genera present in the water samples, SF2 was more diverse, with 87 genera, than SF1 with 76 genera. Overlapping genera were rare, which was an impact of the discharge from FMWWTP between the downstream and upstream samples. In SF1, "Unclassified_f_Euglenaceae" comprised $29.32 \%$ of the eukaryotic genera, $24.62 \%$ belonged to "Unclassified_g_norank_ o_Haplotaxida", $13.47 \%$ to "Unclassified_f_Euglenaceae" 9.61\% to Heteronematina, and $6.86 \%$ to "norank_f_Bacillariophyceae" (Figure 2). Meanwhile, in SF2, $42.42 \%$ were "Unclassified_f_Bdelloidea", $18.78 \%$ were Petalomonas, $13.24 \%$ were 'Unclassified_d_Eukaryota", and $9.87 \%$ were Euplotes. It is well-known that these protozoa play active roles in nutrient removal in aquatic systems, by means of mineralization or by feeding off dispersed bacteria to eliminate the pollution (Pernthaler, 2005). Protists (including flagellates, ciliates, and amoebae) are able to control bacterial growth and alter their diversity, architecture and function (Besemer, 2015).

\subsection{Phylum-Level Bacterial, Eukaryotic, and Fungal Communities and Their Distributions}

A total of 26 bacterial phyla were identified from the water samples. Phylogenetically close phyla were observed to cluster in the SB communities (Figure 1). Among these, the phylum Proteobacteria was evidently abundant in both SB1 (44.18\%) and SB2 (52.11\%). Proteobacteria are widely distributed and often dominate microbial communities within waterbodies. This phylum has a wide variety of members that are associated with nutrient metabolism, $\mathrm{C}$ and $\mathrm{N}$ cycling, and water purification. Therefore, it can be reasonably concluded that the water downstream of the discharge point was more likely to contain more members of the Proteobacteria phylum, including potentially health-threatening members. The discharge from FMWWTP is the connection of sewage to water. Close attention should be paid to the disturbance of the microbial community structure and diversity in the natural waterbody. On the basis of these results and their implications, measures should be taken to improve the wastewater treatment processes to reduce the risks of reusing the water and to uphold responsibility 
for public access.

The phylum Bacteroidetes, which consists of Cytophagia, Flavobacteriia, and Sphingobacteriia, was the second most abundant phylum in both SB1 $(31.31 \%)$ and SB2 (22.72\%). Bacteroidetes are responsible for the degradation of suspended particles consisting of refractory organic material. The infectivity of this phylum alone is distinctly weaker than that of Proteobacteria. The abundances of Nitrospirae, Actinobacteria, Cyanobacteria, Acidobacteria, Chloroflexi, Parcubacteria, Chlorobi, and Planctomycetes in SB2 were greater than in SB1. Among them, the Nitrospirae abundance increased from 0\% (SB1) to $9.76 \%$ (SB2). This suggested that such bacteria that are responsible for nitrogen cycling were more active downstream or in the discharge from the plant. This result is in agreement with the results of many previous studies, both in sediments and

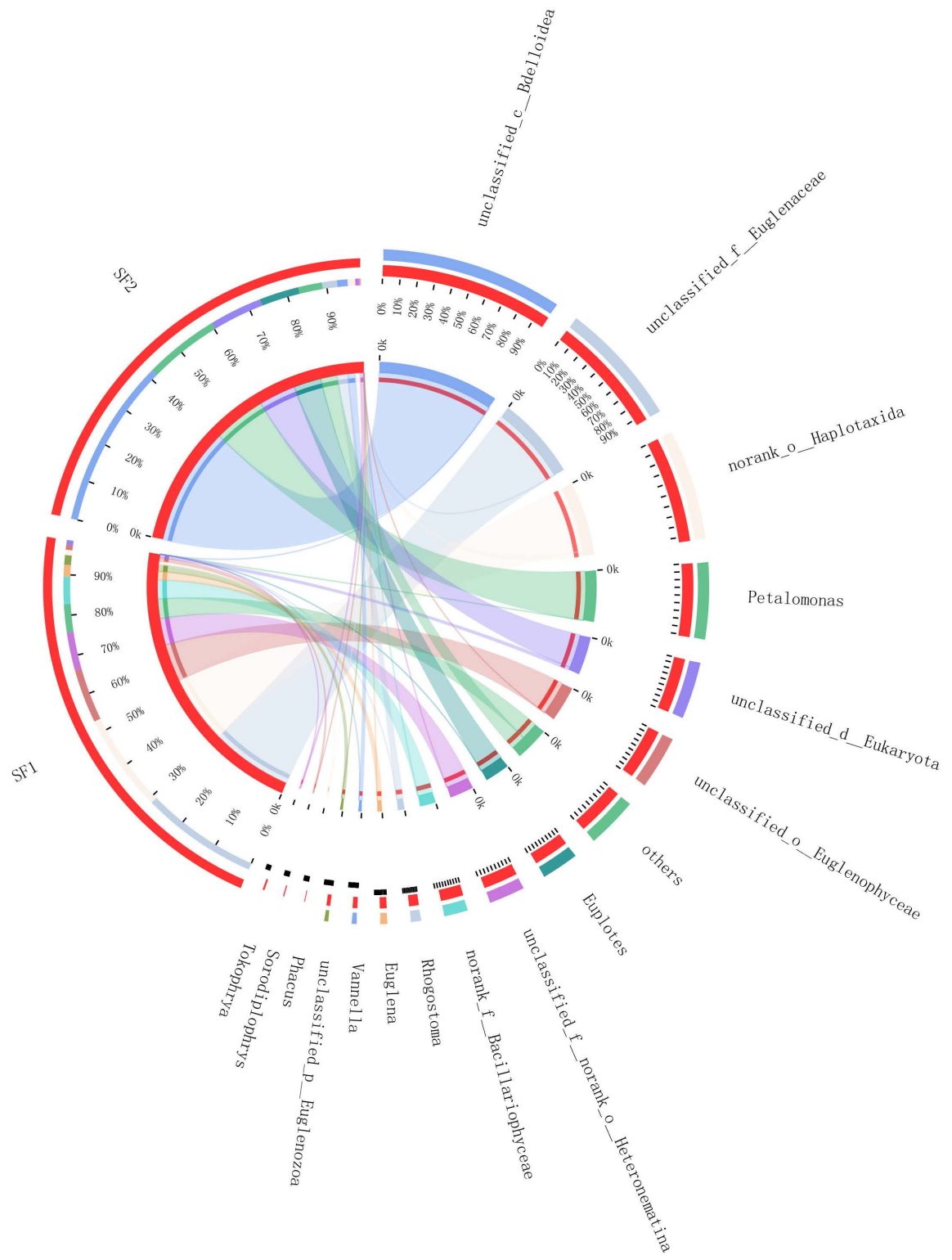

(a) 


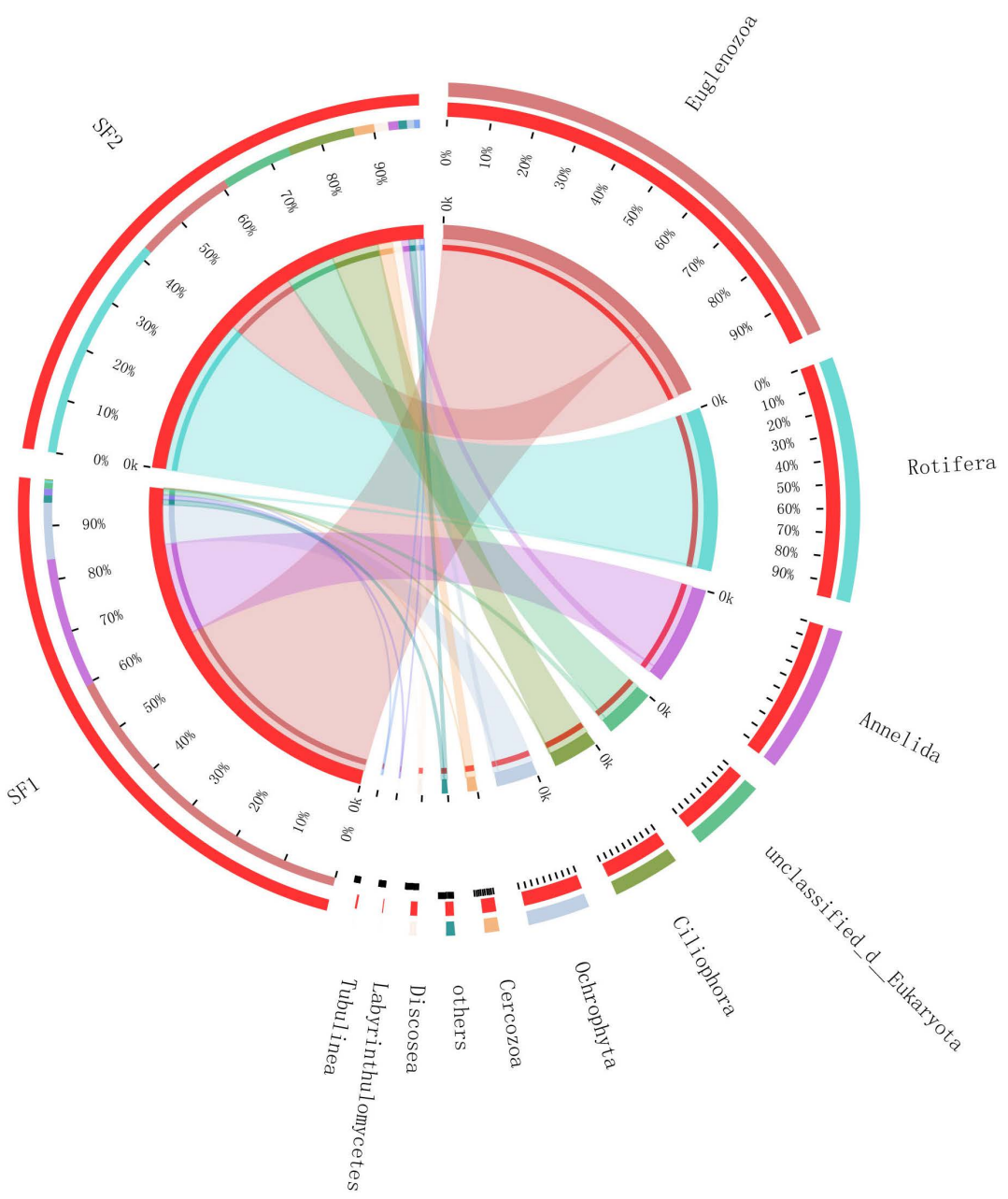

(b)

Figure 2. Eukaryotic microbial community composition, at the genus (a) and phylum-level (b), of samples collected upstream (SF1) and downstream (SF2) of the discharge released from Fuxin Meng Wastewater Treatment Plant into the Xi River.

downstream water. Until now, the FMWWTP has strongly impacted the Xi River microbial community diversity via the heterogeneity of its effluent. Proteobacteria, along with the Cyanobacteria and Actinobacteria found, contained multiple genera that are considered to be available nitrogen and $\mathrm{CO}_{2}$ fixers (Wakelin et al., 2008). Considering this, the wastewater must be treated with the aim of eliminating pollution from nutrient loading.

The eukaryotic microbial community, which is an important and abundant functional element of every aquatic ecosystem, was also influenced by FMWWTP (Figure 2). The eukaryotic microbial diversity of SF2 was lower than that of SF1. The phyla were recognized by using the primers Euk1391F/BR, which tend to have a preference for protists in aquatic environments. In SF1, $60.40 \%$ of the eukaryotic microbial population was affiliated to the Euglenozoa phylum. The fourth and fifth most dominant eukaryotic microbial phyla were Annelida (24.62\%) and Ochrophyta (10.62\%), respectively. In SF2, the abundances of 
Euglenozoa, Annelida, and Ochrophyta were reduced to $19.26 \%, 1.91 \%$, and $1.33 \%$, respectively. In SF2, the abundance of Rotifera was $42.47 \%$ and of Ciliophora was $12.62 \%$, which increased from $0.46 \%$ and $0.19 \%$ in SF1, respectively. This explained the reduction in $\mathrm{BOD}_{5}$ in the downstream sample. Euglenozoa, Annelida, Ochrophyta, Rotifera, and Ciliophora are frequently identified from freshwater sediments and are also used as indicators for biomonitoring (Matsunaga et al., 2014). Rotifera species are regarded as links between the microbial loop and the aquatic food chain (Besemer, 2015). Several studies have shown that protozoa release excretions to bulk solutions and that the nutrients in the excretion may be available for bacteria to utilize for degrading wastes (Matsunaga et al., 2014). Thus, protozoans and metazoans contribute to the mineralization of organic pollutants (More et al., 2010).

The abundance of fungal phyla in the SFF samples was lower than the abundances of phyla in the SB or SF samples. This was due to the fact that the unclassified phyla occupied $87.5 \%$ (SFF1) and $70.8 \%$ (SFF2) of the total phyla in terms of relative abundance (Figure 3). Rozellomycota, the most abundant fungal phylum, had a relative abundance of $6.52 \%$ in SFF1 and of $27.44 \%$ in SFF2. The second dominant phylum was Chytridiomycota, which had a relative abundance of $4.58 \%$ in SFF1 and no identification in SFF2. Chytridiomycota possess active motility via a single flagellum, and can degrade chitin and keratin. Species within this phylum are generally present in ponds, streams, and moist terrestrial habitats. In light of these relative changes in abundance, it was tentatively speculated that water mixing caused by discharge from FMWWTP was of little conse

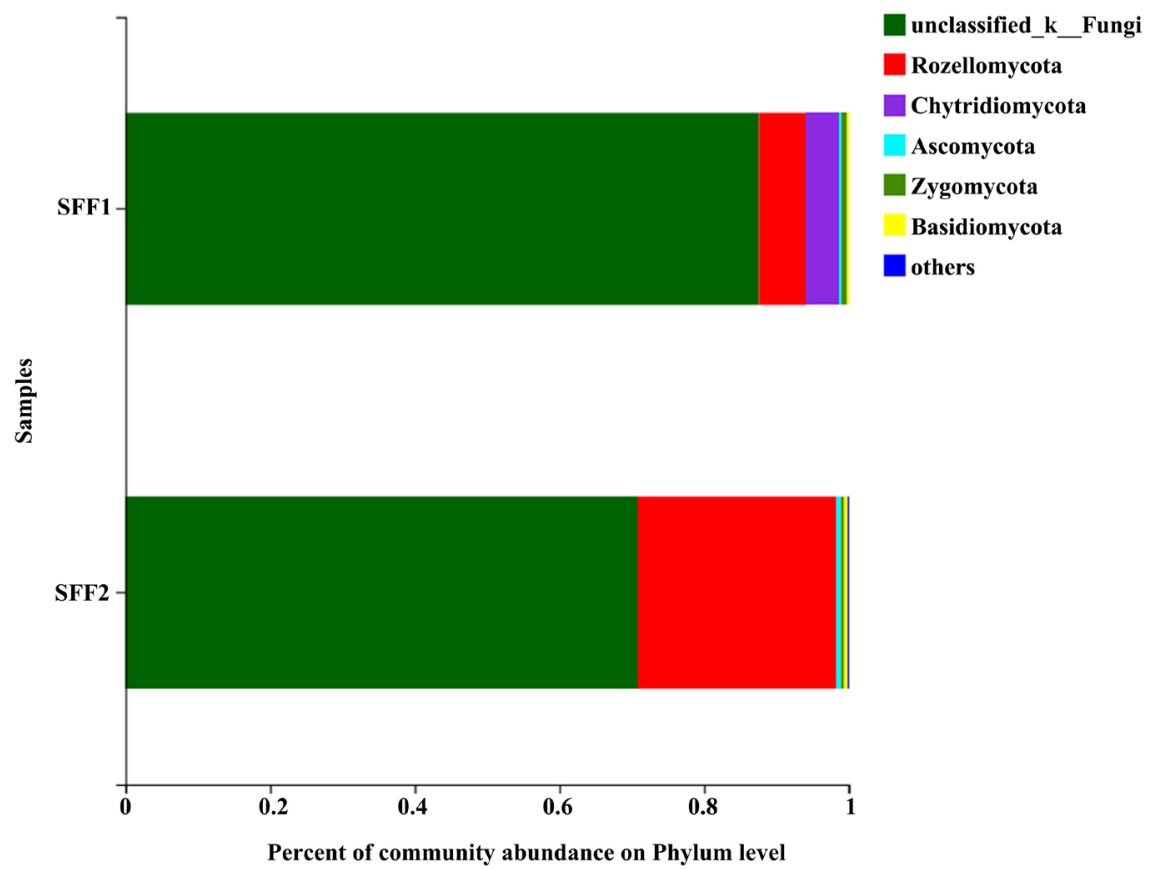

Figure 3. Fungal community composition at the phylum level of samples collected upstream (SFF1) and downstream (SFF2) of the discharge released from Fuxin Meng wastewater treatment plant into the Xi River. 
quence to the fungal community. No further genus data were defined within the fungal phyla.

Fungi, which play an irreplaceable role in water treatment, appear to show a greater ability to degrade organic matter than bacteria, which may be due to their stronger resistance to environmental stress (Prigione et al., 2008). Fungi are more tolerant of pollutants than bacteria, due to their large chromosomes. Furthermore, the fungal cell wall, which consists of an extra-polysaccharide matrix, provides protection against inhibitory compounds via adsorption. A variety of bacteria and fungi may co-metabolize substances simultaneously to achieve a better degradation in the wastewater.

\subsection{The Community Assessment Was Primer-Preferential}

The preferences of the primers were suggested in the process of community component identification. The primer sets, Euk1391F/EukBR and ITS1F/ITS2R, both target eukaryotes from the same sample. According to the data obtained, the primer pair, Euk1391F/EukBR, seems to preferably recognize eukaryotes, such as the protozoans or metazoans of the SF communities. Concomitantly, the ITS1F/ITS2R primer set was effective at characterizing fungi in the SFF samples, which was in accordance with the observations of other previous studies (Guarro et al., 1999; Xu, 2016). Even though the overlapping taxa identified using the two primer pairs were phylogenetically predictable, no crossover taxa were found upon analysis at the various taxon levels. Overall, the choice of primer set directly impacted the accuracy of the community analysis. Therefore, the understanding of the community structures was only primer-specific, which is true for all community assessments. It is necessary to obtain a complete description of the community structure and diversity by combining these results with the results obtained using different universal primer pairs.

\subsection{Community Structures Were Sample-Sensitive}

Fortunately, the same results of the diversity from primer Pairs 27F/338R and ITS1F/ITS2R offset the sensitivity originating from the samples. With regards to the community structures, it is easy to conclude that microbial communities can be regarded as the sentinels and integrators of environmental changes (Ramette, 2007). The insights gained from this work only represented the community structure at the specific sampling sites in the beginning of May and were limited to the current understanding of the evolution of microbial life.

Even the gene library size contributed to the community descriptions. The observed community shifts suggest that the community complexity might be much greater than previously thought between the upstream and downstream samples (Niu et al., 2016). Community analysis data are randomly influenced by the sampling method, sample processing, and environmental factors. The microbial community is well known to be shaped by the interplay of biotic and abiotic interactions (Besemer, 2015; Kumari et al., 2015). The interactions among 
microbes, mycotoxins, and pollutants remain complicated or unclear. In response to environmental factors, the water ecosystem may function as a buffering system for the shift between the rare and abundant strains in the community. Further investigation is required to reveal these interactions. It is believed that a large proportion of microorganisms remain unidentified in the communities when $16 \mathrm{~S}$ and $18 \mathrm{~S}$ rRNA sequencing analysis is used.

This study is helpful for evaluating the safety of reusing water from rivers subjected to treated wastewater discharge, in terms of the prokaryotic and eukaryotic microorganisms present. Microbial community analysis is a prerequisite to the adoption of certain strategies to meet the stipulated sanitary standard. The identification of community members at the phylum and genus levels potentially serves as an indicator of the biochemical properties of the wastewater. Understanding the indigenous microbial community may help to promote river conservation and remediation through chemical, biological, or physical measures.

\section{Conclusion}

In this study, the results of the primer-preferential microbial assessment were generally in line with the biochemical analyses of Xi River. The alpha diversity was higher downstream than the upstream, except for the eukaryotic (SF) samples. Overall, the finding of microbial community assessment of FMWWTP gave side effect to its receiving water at the time. Thus it was necessary to improve the operation of WWTP to live up to the environmental quality standards.

\section{Data Availability}

All of the datasets support the findings of this study are available from the corresponding author $\mathrm{H}$. Zhu upon reasonable request.

\section{Acknowledgements}

We would like to express heartfelt thanks to the excellent anonymous reviewer entrusted by Shanghai Elixigen Company, China. This research is supported by the National Key R\&D Program of China [2019YFC1803800].

\section{Conflicts of Interest}

The authors declare no conflicts of interest regarding the publication of this paper.

\section{References}

Adams, R. I., Miletto, M., Taylor, J. W., \& Bruns, T. D. (2013). Dispersal in Microbes: Fungi in Indoor Air Are Dominated by Outdoor Air and Show Dispersal Limitation at Short Distances. Isme Journal, 7, 1262-1273. https://doi.org/10.1038/ismej.2013.28

Amaral-Zettler, L. A., McCliment, E. A., Ducklow, H. W., \& Huse, S. M. (2009). A Method for Studying Protistan Diversity Using Massively Parallel Sequencing of V9 Hypervariable Regions of Small-Subunit Ribosomal RNA Genes. PLoS ONE, 4, e6372. https://doi.org/10.1371/journal.pone.0006372

Begerow, D., Nilsson, H., Unterseher, M., \& Maier, W. (2010). Current State and Perspec- 
tives of Fungal DNA Barcoding and Rapid Identification Procedures. Applied Microbiology and Biotechnology, 87, 99-108. https://doi.org/10.1007/s00253-010-2585-4

Besemer, K. (2015). Biodiversity, Community Structure and Function of Biofilms in Stream Ecosystems. Research in Microbiology, 166, 774-781. https://doi.org/10.1016/j.resmic.2015.05.006

Blackwood, C. B., Oaks, A., \& Buyer, J. S. (2005). Phylum- and Class-Specific PCR Primers for General Microbial Community Analysis. Applied and Environmental Microbiology, 71, 6193-6198. https://doi.org/10.1128/AEM.71.10.6193-6198.2005

Frigon, D., Biswal, B. K., Mazza, A., Masson, L., \& Gehr, R. (2013). Biological and Physicochemical Wastewater Treatment Processes Reduce the Prevalence of Virulent Escherichia coli. Applied and Environmental Microbiology, 79, 835-844.

https://doi.org/10.1128/AEM.02789-12

Ghilamicael, A. M., Boga, H. I., Anami, S. E., Mehari, T., \& Budambula, N. L. M. (2018). Potential Human Pathogenic Bacteria in Five Hot Springs in Eritrea Revealed by Next Generation Sequencing. PLoS ONE, 13, e0194554.

https://doi.org/10.1371/journal.pone.0194554

Guarro, J., Gene, J., \& Stchigel, A. M. (1999). Developments in Fungal Taxonomy. Clinical Microbiology Reviews, 12, 454-500. https://doi.org/10.1128/CMR.12.3.454

Huang, Z., Gedalanga, P. B., Asvapathanagul, P., \& Olson, B. H. (2010). Influence of Physicochemical and Operational Parameters on Nitrobacter and Nitrospira Communities in an Aerobic Activated Sludge Bioreactor. Water Research, 44, 4351-4358. https://doi.org/10.1016/j.watres.2010.05.037

Kumari, P., Choi, H. L., \& Sudiarto, S. I. A. (2015). Assessment of Bacterial Community Assembly Patterns and Processes in Pig Manure Slurry. PLoS ONE, 10, e0139437. https://doi.org/10.1371/journal.pone.0139437

Matsunaga, K., Kubota, K., \& Harada, H. (2014). Molecular Diversity of Eukaryotes in Municipal Wastewater Treatment Processes as Revealed by 18S rRNA Gene Analysis. Microbes and Environments, 29, 401-407. https://doi.org/10.1264/jsme2.ME14112

More, T. T., Yan, S., Tyagi, R. D., \& Surampalli, R. Y. (2010). Potential Use of Filamentous Fungi for Wastewater Sludge Treatment. Bioresource Technology, 101, 7691-700. https://doi.org/10.1016/j.biortech.2010.05.033

Niu, J., Deng, J., Xiao, Y., He, Z., Zhang, X., Van Nostrand, J. D. et al. (2016). The Shift of Microbial Communities and Their Roles in Sulfur and Iron Cycling in a Copper Ore Bioleaching System. Scientific Reports, 6, Article No. 34744.

https://doi.org/10.1038/srep34744

Pernthaler, J. (2005). Predation on Prokaryotes in the Water Column and Its Ecological Implications. Nature Reviews Microbiology, 3, 537-546.

https://doi.org/10.1038/nrmicro1180

Pjevac, P., Schauberger, C., Poghosyan, L., Herbold, C. W., van Kessel, M., Daebeler, A. et al. (2017). AmoA-Targeted Polymerase Chain Reaction Primers for the Specific Detection and Quantification of Comammox Nitrospira in the Environment. Frontiers in Microbiology, 8, 1508. https://doi.org/10.3389/fmicb.2017.01508

Prigione, V., Tigini, V., Pezzella, C., Anastasi, A., Sannia, G., \& Varese, G. C. (2008). Decolourisation and Detoxification of Textile Effluents by Fungal Biosorption. Water Research, 42, 2911-2920. https://doi.org/10.1016/j.watres.2008.03.003

Ramette, A. (2007). Multivariate Analyses in Microbial Ecology. FEMS Microbiology Ecology, 62, 142-160. https://doi.org/10.1111/j.1574-6941.2007.00375.x

Rapala, J., Niemela, M., Berg, K. A., Lepisto, L., \& Lahti, K. (2006). Removal of Cyanobacteria, Cyanotoxins, Heterotrophic Bacteria and Endotoxins at an Operating Surface 
Water Treatment Plant. Water Science and Technology, 54, 23-28.

https://doi.org/10.2166/wst.2006.443

Ravel, J., Gajer, P., Abdo, Z., Schneider, G. M., Koenig, S. S., McCulle, S. L. et al. (2011). Vaginal Microbiome of Reproductive-Age Women. Proceedings of the National Academy of Sciences of the United States of America, 108, 4680-4687. https://doi.org/10.1073/pnas.1002611107

Reblova, M., Untereiner, W. A., \& Reblova, K. (2013). Novel Evolutionary Lineages Revealed in the Chaetothyriales (Fungi) Based on Multigene Phylogenetic Analyses and Comparison of Its Secondary Structure. PLoS ONE, 8, e63547. https://doi.org/10.1371/journal.pone.0063547

Shi, X., Huang, S., Yeap, T. S., Ong, S. L., \& Ng, H. Y. (2020). A Method to Eliminate Bromide Interference on Standard COD Test for Bromide-Rich Industrial Wastewater. Chemosphere, 240, Article ID: 124804.

https://doi.org/10.1016/j.chemosphere.2019.124804

Wakelin, S. A., Colloff, M. J., \& Kookana, R. S. (2008). Effect of Wastewater Treatment Plant Effluent on Microbial Function and Community Structure in the Sediment of a Freshwater Stream with Variable Seasonal Flow. Applied and Environmental Microbiology, 74, 2659-2668. https://doi.org/10.1128/AEM.02348-07

Wang, F., Men, X., Zhang, G., Liang, K., Xin, Y., Wang, J. et al. (2018). Assessment of 16S rRNA Gene Primers for Studying Bacterial Community Structure and Function of Aging Flue-Cured Tobaccos. AMB Express, 8, 182.

https://doi.org/10.1186/s13568-018-0713-1

Xu, J. P. (2016). Fungal DNA Barcoding, Genome, 59, 913-932. https://doi.org/10.1139/gen-2016-0046

Ye, F., \& Kameyama, S. (2020). Long-Term Spatiotemporal Changes of 15 Water-Quality Parameters in Japan: An Exploratory Analysis of Countrywide Data during 1982-2016. Chemosphere, 242, Article ID: 125245. https://doi.org/10.1016/j.chemosphere.2019.125245

Yin, H., Zhang, X., Li, X., He, Z., Liang, Y., Guo, X. et al. (2014). Whole-Genome Sequencing Reveals Novel Insights into Sulfur Oxidation in the Extremophile Acidithiobacillus thiooxidans. BMC Microbiology, 14, 179. https://doi.org/10.1186/1471-2180-14-179

Yotova, G., Lazarova, S., Kudlak, B., Zlateva, B., Mihaylova, V., Wieczerzak, M. et al. (2019). Assessment of the Bulgarian Wastewater Treatment Plants' Impact on the Receiving Water Bodies. Molecules, 24, 2274. https://doi.org/10.3390/molecules24122274

Zhao, Y., Fang, Y., Jin, Y., Huang, J., Bao, S., Fu, T. et al. (2014). Potential of Duckweed in the Conversion of Wastewater Nutrients to Valuable Biomass: A Pilot-Scale Comparison with Water Hyacinth. Bioresource Technology, 163, 82-91.

https://doi.org/10.1016/j.biortech.2014.04.018 